\title{
Inspection duration and the linear motion aftereffect: Preliminary report
}

\author{
MAURICE HERSHENSON \\ Brandeis University, Waltham, Massachusetts
}

\begin{abstract}
Two subjects rated the strength of motion of the linear motion aftereffect (MAE) produced by a horizontal grating following inspection periods of $0.5,1,5,10$, and $15 \mathrm{~min}$. There was only one trial per day. The MAE consisted of three phases: an initial maximum-strength phase, a decay phase, and a tail. The duration of all three phases increased and the decay rate decreased with increasing inspection duration. The decay time constant increased as a linear function of inspection duration.
\end{abstract}

Although motion aftereffects (MAEs) have been studied extensively (Anstis, 1984; Beverley \& Regan, 1979; Bonnet, 1978; Holland, 1965; Sekuler, 1975; Wohlgemuth, 1911), the role of inspection duration remains unclear. Early studies reported that the duration and time constant of the MAE increased wtih increasing inspection duration up to 5 min (Bakan \& Mizusawa, 1963; Eysenck \& Holland, 1960; Holland \& Eysenck, 1960; Taylor, 1963). In contrast, recent studies have found that a plateau is reached much sooner. For example, Bonnet (1973) found that the duration of the MAE produced by a rotating spiral reached a plateau of about $13 \mathrm{sec}$ after 25-30 sec of adaptation. Lehmkuhle and Fox (1975) found that the duration of the MAE produced by the motion of a vertical grating increased as a linear function of inspection duration up to about $60 \mathrm{sec}$ and then leveled off. Sekuler (1975) reported that direction-specific thresholds measured after a moving horizontal grating was viewed increased with inspection duration, but reached a plateau after about $100 \mathrm{sec}$ of adaptation. Additional observations using inspection durations of 35-45 min yielded threshold values nearly identical to that for $100 \mathrm{sec}$ of adaptation. Sekuler noted, however, that recovery from protracted exposures required extra time, an observation that suggests longer time constants.

Although the results of the recent studies are consistent, there are differences among the studies that raise questions. The earlier studies measured responses to rotating spirals or, in the case of Taylor (1963), to a rotating disc "marked with an irregular swirling pattern in red pencil"' (p. 120). In light of recent evidence that the visual system responds to components of complex proximal motion patterns (Hershenson, 1987) and that these responses may be mediated by different neural structures whose recovery durations and time constants differ (Beverley \& Regan, 1979; Cavanagh \& Favreau, 1980; LonguetHiggins \& Prazdny, 1980; Regan, 1986; Regan \& Bever-

I thank Steven P. Schwartz for collecting the data. Requests for reprints should be sent to Maurice Hershenson, Department of Psychology, Brandeis University, Waltham, MA 02254. ley, 1978), it is not clear what component of the MAE was being measured or whether the components are comparable across studies using qualitatively different stimuli.

In the more recent studies, Bonnet (1973) tested inspection durations up to only $35 \mathrm{sec}$. Lehmkuhle and Fox (1975) described only one observation for each inspection duration for each subject. Furthermore, their data show different relationships for the 3 subjects at the longer inspection durations. MAE durations apparently leveled off at $40 \mathrm{sec}$ of inspection duration for 2 of the subjects, but for 1 of them it apparently increased again after $80 \mathrm{sec}$. MAE duration apparently rose continuously up to $80 \mathrm{sec}$ of inspection duration for the 3rd subject. The data reported by Sekuler (1975), although convincing, describe direction-specific threshold changes, not the duration and time constant of the MAE. These temporal characteristics of the MAE may be related in the same way to inspection duration, but it is better to measure the relationship directly than to assume that one exists.

The present experiment assessed the effect of inspection duration on the duration and time constant of the linear MAE. Before the description of the experiment, a discussion of the method of measurement is in order. Measurements of MAEs frequently involve matching or nulling procedures. For example, Taylor's (1963) subjects continuously adjusted the speed of rotation of the test stimulus so that the disc appeared stationary, a procedure that necessarily took time. The rotation speed that was required to eliminate the MAE was the dependent variable. Regan and Beverley (1978) used a nulling method along with the method of adjustments. Settings were made in 15-sec intervals with $1 \mathrm{~min}$ of readaptation between settings.

There are two reasons why these methods were not used in this experiment. First, the strength of the MAE declines rapidly. Even the relatively short time it takes to make an adjustment can correspond to a large loss in MAE strength. To avoid this problem, a magnitude estimation procedure was used, whereby subjects rated the strength of the MAE by continuously calling out numbers between 10 and 0 . Second, the attempt to compensate for the loss 
in MAE strength during measurement by introducing an additional inspection period after each setting makes it impossible to determine the effect of a specific inspection duration or to evaluate the role of the additional periods. To avoid this problem and to minimize the possible additive effects of repeated exposures to the moving inspection stimulus (Hershenson, 1985; Kalfin \& Locke, 1972; Masland, 1969), only one trial was run on a given day. Thus, a minimum of $22 \mathrm{~h}$ separated trials throughout the experiment.

\section{METHOD}

\section{Subjects}

There were 2 subjects, the author (M.H.) and an undergraduate student (I.L.). Both practiced using the rating scale on rotation MAE (described below) in two 1-h sessions.

\section{Stimulus}

The stimulus was a horizontal square-wave grating (spatial frequency approximately 3.2 cycles per degree), consisting of alternating black (India ink) and white bars on a continuous paper loop. The bars and spaces subtended approximately $0.2^{\circ}$ of visual angle. The grating moved downward at a rate of 6 cycles per second and was visible within a square aperture that measured approximately $4.5^{\circ}$ of visual angle on a side. The stimulus field was illuminated by overhead fluorescent lights and a projector lamp focused on the surface of the stimulus. The illumination falling on the stimulus was approximately $1.4 \mathrm{~lx}$.

\section{Procedure}

There were five inspection durations: $0.5,1,5,10$, and $15 \mathrm{~min}$. Only one test was performed on a given day, allowing a minimum of $22 \mathrm{~h}$ between tests. One series consisted of tests using all the inspection durations. Within series, the order of inspection duration was selected randomly. Each subject participated in three series.

The subjects fixated the center of the aperture monocularly with the right eye. They were informed of the passage of inspection time at the 10-, 5-, 2-, and 1-min marks and at 30 and $15 \mathrm{sec}$. With $10 \mathrm{sec}$ remaining in the inspection period, the experimenter marked each remaining second. When the motion of the grating stopped at the end of the inspection period, the subjects maintained fixation at the center of the grating. They rated the strength of the MAE continuously using an 11-point scale, where 10 represented the strength of perceived motion of the MAE immediately upon cessation of the motion of the inspection stimulus, and 0 represented no perceptible motion. Note that 10 described the initial perceived strength of motion of the MAE. Consequently, the scale did not index absolute strength. Ratings were recorded every $5 \mathrm{sec}$. The subjects stopped rating the MAE motion when it was rated " 0 "' on two consecutive vocalizations.

\section{RESULTS AND DISCUSSION}

\section{Qualitative Description of the Linear MAE}

A protocol for a typical trial began with MAE strength's being rated " 10 " immediately upon cessation of stimulus motion. It continued with a succession of decreasing strength ratings, and concluded with either a single " 1 " rating or a succession of " 1 " ratings. Qualitative differences in the protocols were obvious at both ends of the scale. At the high end, the strength of the MAE was reported to remain at maximum for a period of time, following some of the longer inspection durations. Specifically, after $15 \mathrm{~min}$ of inspection, both subjects continued to rate the strength of the MAE as " 10 " after $5 \mathrm{sec}$ in one trial, and 1 subject (I.L.) continued to rate it " 10 " after $10 \mathrm{sec}$ in two trials. For this subject, MAE strength was also rated " 10 " after $5 \mathrm{sec}$ in one trial following $10 \mathrm{~min}$ of inspection and in two trials following $5 \mathrm{~min}$ of inspection. At the low end of the scale, 1 subject (M.H.) ended all ratings with a single " 1 " rating. For the other subject, the number of " 1 " ratings increased with increasing inspection duration: $3,5,7,6$, and 13 for inspection durations of $0.5,1,5,10$, and $15 \mathrm{~min}$, respectively.

Qualitatively, the protocols suggest three distinct phases to the linear MAE: an initial maximum-strength phase, a decay phase, and a tail. The latter two phases correspond to those reported by Taylor (1963). In the initial phase, the rated strength of the MAE remained at maximum for a period of time. This phase was not evident in MAEs produced by shorter inspection durations, either because the MAE began to decay immediately or because the MAE decayed rapidly enough to make the rating less than " 10 ", at the 5-sec mark. In general, the longer the inspection duration, the longer the rated strength of the MAE remained at maximum.

Regardless of the duration of the initial maximumstrength phase, the decay phase manifested an exponential decrease in the rated strength of the MAE (see below). The final phase of the MAE was a tail, manifested as a period in which the strength was rated "1." This phase lasted up to $20 \mathrm{sec}$ for MAEs produced by longer durations of inspection.

\section{Quantitative Description of the Linear MAE}

The duration of the MAE was defined as the last 5-sec interval in which the strength was rated " 1 ." Table 1 shows the mean (geometric) duration of the MAE as a function of inspection duration for each subject. Overall, duration increased with inspection duration, but the rates of increase were different for the 2 subjects.

The decay phase of the linear MAE was defined as that portion of the protocol between the last reported " 10 " and the first reported " 1 ," inclusive. A least squares regression line was fit to the logarithm of the strength ratings, plotted as a function of time for each trial. Each regression line yielded a time constant and an intercept for each trial. Analyses of variance (ANOVAs) were computed separately on log transforms of the time constants and intercepts. The time constants produced by the 2 subjects differed $[F(1,4)=97.51, p<.01]$, but the intercepts did not $[F(1,4)<1]$. Interactions were not significant $\left[F_{\mathrm{s}}(4,16)=1.19\right.$ and $1.61, p>.05$, for time constants and intercepts, respectively]. Intercepts did not

Table 1

Mean Duration of the Linear Motion Aftereffect (in sec) for Five Inspection Durations for 2 Subjects $(n=3)$

Inspection Duration (in min)

\begin{tabular}{llllcc}
\cline { 2 - 6 } Subject & 0.5 & 1 & 5 & 10 & 15 \\
\hline M.H. & 20 & 20 & 30 & 29.7 & 33.3 \\
I.L. & 28.2 & 42.9 & 59.5 & 66.4 & 83.1 \\
\hline
\end{tabular}


produce a significant main effect $[F(4,16)=1.68$, $p>.05]$. This is not surprising because, as noted earlier, the upper end of the rating scale is indexed to the initial strengths of the MAE on that trial.

The major effect was clear: the time constant increased as a linear function of inspection duration $[F(1,6)=$ $157.84, p<.01]$ for the linear trend component, accounting for $96 \%$ of the variance due to inspection duration. This increase can be seen in Table 2 , which gives the mean (geometric) decay time constant and standard error for each inspection duration for each subject. It is not obvious, however, that the time constants increased for the longer durations. To check this, a second ANOVA was computed using only those entries for the 5-, 10-, and 15-min inspection durations. Once again, the subjects differed in their overall values $[F(1,4)=42.01$, $p<.01]$, but the subject $\times$ condition interaction was not significant $[F(2,8)=1.44, p>.05]$. The linear trend component of the inspection duration main effect was significant $[F(1,8)=21.04, p<.01]$, accounting for $86 \%$ of the variance. Therefore, it is reasonable to conclude that the time constant was a linear increasing function of inspection duration for durations up to $15 \mathrm{~min}$.

The increase in time constant with increasing inspection duration could have been caused by a couple of factors. One possibility is that the duration of the initial phase increased with inspection duration, a possibility supported by the protocols. A second possibility is that, as suggested by Sekuler (1975), the decay occurred more slowly following longer inspection durations. To examine this possibility, least squares regression lines were fit to the log strength ratings over time for each subject using all trials at each inspection duration. These lines are shown in Figure 1. The proportion of variance accounted for by each line is given in parentheses. Both subjects showed the same systematic change: MAE strength declined less rapidly with increasing inspection duration.

To summarize, the strength ratings of the linear MAE manifested three distinct phases: an initial maximumstrength phase, a decay phase, and a tail. The duration of all three phases increased with increasing inspection duration. The decay time constant increased as a linear function of inspection duration.

The most important immediate question to ask is whether the data can be attributed to a methodological artifact or to response bias. Although these possibilities can never be ruled out with certainty, their likelihood is

Table 2

Mean Decay Time Constants (DTCs) (in sec) and Standard Errors (SEs) for Five Inspection Durations for 2 Subjects $(n=3)$

\begin{tabular}{cccccc} 
& \multicolumn{4}{c}{ Subject } \\
\cline { 2 - 3 } $\begin{array}{c}\text { Inspection } \\
\text { Duration (in min) }\end{array}$ & DTC & $S E$ & & DTC & $S E$ \\
\hline 0.5 & 12.7 & 5.1 & & 15.8 & 3.8 \\
1 & 11.2 & 3.2 & 23.5 & 4.3 \\
5 & 15.1 & 3.8 & 27.6 & 4.8 \\
10 & 13.7 & 4.1 & 30.5 & 5.6 \\
15 & 16.4 & 4.1 & 39.2 & 5.8 \\
\hline
\end{tabular}

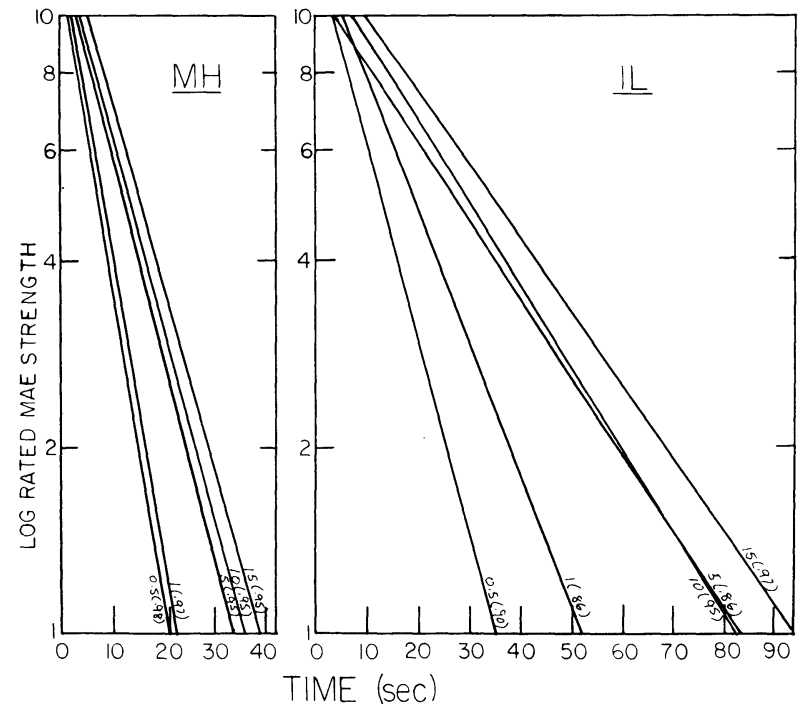

Figure 1. Least squares regression lines fit to log strength ratings over time for decay portions of linear motion aftereffects (MAEs) for five inspection durations. The proportion of the variance accounted for by the line is given in parentheses. MH and IL were the subjects.

diminished by the fact that only a single inspection duration was tested on a single day. The artifact or biasing factor would have to carry over trials separated by at least $22 \mathrm{~h}$, and in some cases $72 \mathrm{~h}$. Moreover, it would have to be precise enough to generate a linear increase in the time constant with inspection duration, a relationship that accounted for $96 \%$ of the variance.

The subjects, however, were aware of the range of inspection durations that were to be used and possibly "expected" to see longer lasting MAEs following longer inspection periods. But it is unlikely that this kind of expectancy could have produced the precise results described above, because, first, the order of inspection duration was randomized within series; second, three series were presented; and third, only one inspection duration was tested on a given day.

It is also possible that the report of a maximum-strength initial phase was an artifact of the rating scale used, which may have forced an artificial limit on the sensitivity of the subjects at the extremes. Consequently, they may not have noticed the slight decrease in strength that occurred immediately upon cessation of the motion of the stimulus. This explanation also seems unlikely, because the subjects began calling out numbers immediately and continued over time. There was no absolute initial decrement that had to be reached before a rating of " 9 " and it is unlikely that the subjects anticipated a future perception in their momentary rating of the strength of the MAE. The subjects were completely free to respond as soon as the slightest decrement was noticed. It seems reasonable to assume, therefore, that they did so.

The differences between this experiment and previous assessments of the role of inspection duration are obvious. The studies differed either in the stimulus used to 
produce the MAE (spirals or rotating lines) or in the response used to measure the effect of looking at a moving grating for a long period of time. With respect to the stimulus differences, it is likely that the visual system responds to complex motion patterns by extracting orthogonal components (Hershenson, 1987), and it is not clear how the MAEs produced by those components are related to the linear MAEs measured in this experiment. With respect to the response differences, it is possible that threshold and superthreshold phenomena are affected differently by inspection duration.

Clearly, it is not possible to generalize from measurements using a single grating at a single luminance level. Nevertheless, if the data provide an accurate description of the effect of inspection duration on the strength of the linear MAE, one can ask what they imply about our conception of the mechanisms that produce the MAE. First, one can safely say that the mechanism is more complex than has been proposed; it has usually been assumed that a recovery begins immediately (Barlow \& Hill, 1963; Keck \& Pentz, 1977; Sekuler, 1975; Sekuler \& Pantle, 1967; Sutherland, 1961). Moreover, most researchers assume that the time constant is independent of inspection duration and that the time constant can be used to characterize the underlying structure (Regan \& Beverley, 1978). The data reported here suggest that our understanding of the mechanism underlying the MAE requires reevaluation.

\section{REFERENCES}

ANSTIS, S. (1984). Aftereffects of form, motion, and color. In L. Spillman \& B. R. Wooten (Eds.), Sensory experience, adaptation, and perception (pp. 583-601). Hillsdale, NJ: Erlbaum.

BaKan, P., \& Mizusawa, K. (1963). Effects of inspection time and direction of rotation on a generalized form of the spiral aftereffect. Journal of Experimental Psychology, 65, 583-586.

Barlow, H. B., \& HiLl, R. M. (1963). Evidence for a physiological explanation of the waterfall phenomenon and figural aftereffects. $\mathrm{Na}$ ture (London), 200, 1345-1347.

BeVERLEY, K. I., \& ReGAN, D. (1979). Separable aftereffects of changing-size and motion-in-depth: Different neural mechanisms? Vision Research, 19, 727-732.
BoNNET, C. (1973). Facteurs temporels dans le mouvement consécutif visuel. Vision Research, 13, 1311-1317.

BoNNET, C. (1978). Time factors in the processing of visual movement information. In J. Requin (Ed.), Attention and performance VII (pp. 2541). Hillsdale, NJ: Erlbaum.

Cavanagh, P., \& Favreau, O. E. (1980). Motion aftereffect: A global mechanism for the perception of rotation. Perception, 9, 175-182.

EysENCK, H. J., \& HollaND, H. (1960). Length of spiral after-effect as a function of drive. Perceptual \& Motor Skills, 11, 129-130.

HERSHENSON, M. (1985). Thirty seconds of adaptation produce spiral aftereffects three days later. Bulletin of the Psychonomic Society, 23, 122-123.

Hershenson, M. (1987). Visual system responds to rotational and sizechange components of complex proximal motion patterns. Perception \& Psychophysics, 42, 60-64.

Holland, H. C. (1965). The spiral after-effect. Oxford, England: Pergamon.

Holland, H., \& EySENCK, H. J. (1960). Spiral after-effect as a function of length of stimulation. Perceptual \& Motor Skills, 11, 228.

KALFIN, K., \& LocKE, S. (1972). Evaluation of long-term visual afterimage following monocular stimulation. Vision Research, 12, 359-361.

KeCK, M. J., \& Pentz, B. (1977). Recovery from adaptation to moving gratings. Perception, 6, 719-725.

LEHMKUHLE, S. W., \& Fox, R. (1975). Effect of binocular rivalry suppression on the motion aftereffect. Vision Research, 15, 855-859.

Longuet-Higgins, H. C., \& Prazdny, K. (1980). The interpretation of a moving retinal image. Proceedings of the Royal Society of London, Series $B, 208,385-397$.

MASLAND, R. H. (1969). Visual motion perception: Experimental modification. Science, 165, 819-821.

REGAN, D. (1986). Visual processing of four kinds of relative motion. Vision Research, 26, 127-145.

Regan, D., \& BeverLey, K. I. (1978). Illusory motion in depth: Aftereffect of adaptation to changing size. Vision Research, 18, 209-212.

Sekuler, R. (1975). Visual motion perception. In E. C. Carterette \& M. P. Friedman (Eds.), Handbook of perception: Vol. V. Seeing (pp. 387-433). New York: Academic Press.

Sekuler, R., \& Pantle, A. (1967). A model for the after-effects of seen movement. Vision Research, 7, 427-439.

SuTHERLAND, N. S. (1961). Figural aftereffects and apparent size. Quarterly Journal of Experimental Psychology, 13, 222-228.

TAYLOR, M. M. (1963). Tracking the decay of the aftereffect of seen rotary movement. Perceptual \& Motor Skills, 16, 119-129.

Wohlgemuth, A. (1911). On the after-effect of seen movement. British Journal of Psychology, Monograph Supplement No. 1.

(Manuscript received for publication October 17, 1987.) 